\title{
The variable clinical course of peripartum cardiomyopathy
}

\author{
Jan Krejcia, Petr Hude ${ }^{a}$, Lenka Spinarova ${ }^{a}$, Vita Zampachova ${ }^{b}$, Alzbeta Sirotkovab ${ }^{b}$, Tomas Freibergerc, Eva Nemcovac, \\ Jiri Vitovec ${ }^{\mathrm{a}}$
}

Background. In Europe, peripartum cardiomyopathy (PPCM) is a rare disorder, often difficult to diagnose and it has a variable clinical course. The aim of this report was to describe and discuss the individual variability of this disorder and its management.

Patients and Methods. Three cases of PPCM manifesting as severe heart failure are compared. Common was the presence of myocardial inflammation detected by endomyocardial biopsy. Different were treatment methods and clinical course. Modern therapeutic concepts such as immunosuppressive therapy and bromocriptin administration are discussed, as well as non-pharmacological approaches.

Conclusion. In the differential diagnostics of dyspnea associated with pregnancy and childbirth, PPCM should be considered. The potentially severe course of the disease requires hospitalization with the possibility of comprehensive heart failure treatment, including non-pharmacological approaches such as device therapy and heart transplantation.

Key words: peripartum cardiomyopathy, myocarditis, echocardiography, endomyocardial biopsy, therapy

Received: February 14, 2012; Accepted: July 20, 2012; Available online: October 31, 2012 http://dx.doi.org/10.5507/bp.2012.080

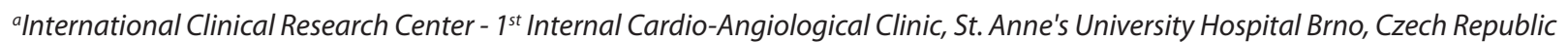
${ }^{b} 7^{\text {st }}$ Department of Pathologic Anatomy, St. Anne's University Hospital and Masaryk University, Brno 'Center of Cardiovascular and Transplant Surgery, Brno

Corresponding author: Jan Krejci, e-mail:jan.krejci@fnusa.cz

\section{INTRODUCTION}

Peripartum cardiomyopathy (PPCM) is a disorder with no clear cause, characterized by systolic dysfunction of the left ventricle (LV) that appears toward the end of pregnancy or during the first months postpartum in women without any previous history of heart failure. This definition was published as a position statement of the Heart Failure Association of the European Society of Cardiology Working Group on peripartum cardiomyopathy in 2010, and is more general than some earlier definitions ${ }^{1}$. A previous definition defined the period of PPCM onset more specifically as within the last month before and the first 5 months after birth ${ }^{2}$. In the new definition, left ventricular dilatation is not a condition for the diagnosis, and systolic dysfunction is interpreted more flexibly - left ventricle ejection fraction (LVEF) should be nearly always below $45 \%$. It is emphasized that PPCM is a diagnosis of exclusion and it is always necessary to exclude other possible causes of heart failure that may occur in the peripartum period (congenital heart disease, primary valvulopathy, coronary artery disease, or other pre-existing heart diseases). The diagnosis is usually (around $80 \%$ of cases) determined after the birth; it is seen less frequently toward the end of pregnancy ${ }^{1,3,4}$.

The first descriptions of heart failure associated with pregnancy appeared in the 19 th century. The term peripartum cardiomyopathy was used for the first time by Demakis in 1971 (ref. $^{3}$ ). The incidence of PPCM in the U.S. is estimated at 1: 2000-4000 live births, and is clearly more common among African-Americans ${ }^{1,3,5}$.
PPCM is included in non-genetic and non-familial forms of dilated cardiomyopathies according to the European Society of Cardiology and the American Heart Association classifications ${ }^{6,7}$. Despite this, it has been observed that PPCM is seen more frequently in some families and frequently occurs in women with a higher familial incidence of dilated cardiomyopathy (DCM). This suggests that PPCM could be part of wider DCMlike-disorder group ${ }^{8,9}$.

The contributing risk factors leading to PPCM include race with a clearly higher incidence and more severe course in afro-american women, higher maternal age, multiple pregnancies, obesity, preeclampsia, hypertension, diabetes, infection, and prolonged use of beta-agonist tocolytics. A familial clustering of PPCM was recently described; however, according to Elkayam et al, the number of previous pregnancies has not been demonstrated as a risk $^{9-11}$.

The clinical course of PPCM corresponds to the presence of systolic heart failure and is dominated by exertional dyspnea or even resting symptoms, often with a picture of paroxysmal nocturnal dyspnea, fatigue, swelling, and weakness. The fact that many of these symptoms could be observed in a significant number of pregnant women without heart failure, especially in the late stages of pregnancy, can lead to the underestimation of symptoms. The possible presence of PPCM should be considered in terms of the strikingly rapid development of symptoms, as well as their intensity. The examination may reveal significant jugular venous dilation, hepatomegaly, edema, pulmonary crackles, gallop, and systolic murmur at the apex ${ }^{10}$. 
The most important method for determining diagnosis is echocardiography. This examination shows dysfunction and possibly dilatation of the LV. Cardiac magnetic resonance imaging (MRI) is also very useful, as it can assess $\mathrm{LV}$ function and detect the presence of inflammation in the myocardium (myocarditis). In this case, endomyocardial biopsy (EMB), which is currently the gold standard for myocarditis diagnosis ${ }^{12-14}$, should be considered and eventually performed. Electrocardiography (ECG) is not very useful for PPCM diagnosis, due to the nonspecific changes accompanying this disorder.

PPCM prognosis remains serious, with 6-month mortality rate ranging between $10-20 \%$, and 2-year mortality around $30 \%$. Mortality varies geographically and especially among different ethnic groups ${ }^{9}$.

Normalization of LV systolic function takes place in $23-41 \%$ of diagnosed patients, and according to some authors in as many as half of the patients at the 6-month follow-up visit ${ }^{15}$. In the U.S., about $10 \%$ of patients undergo heart transplantation. Very low LVEF (below 30\%), as well as LV dilatation (end-diastolic LV dimension more than $60 \mathrm{~mm}$ ), are associated with a significantly higher risk of persistent left ventricular dysfunction ${ }^{1,9,10}$.

\section{CLINICAL CASE 1}

A 24 year old women presented with severe shortness of breath at the $32^{\text {nd }}$ week of pregnancy. This was her first and multiple pregnancy (twins). Dyspnea was discovered about 2 weeks after viral infection and quickly progressed to resting and nocturnal dyspnea. Echocardiographic examination showed severe LV dysfunction (LVEF 15\%) with significant LV dilatation (diastolic diameter $72 \mathrm{~mm}$ ). After stabilization with intravenous diuretic therapy and inotropic circulation support (dobutamine), a Cesarean section was performed in the 34 th week of pregnancy. Both children were born healthy. Despite a complex treatment for heart failure (angiotensin converting enzyme inhibitors, diuretics, spironolactone, and levosimendan), the mother had a severe acute heart failure bordering on

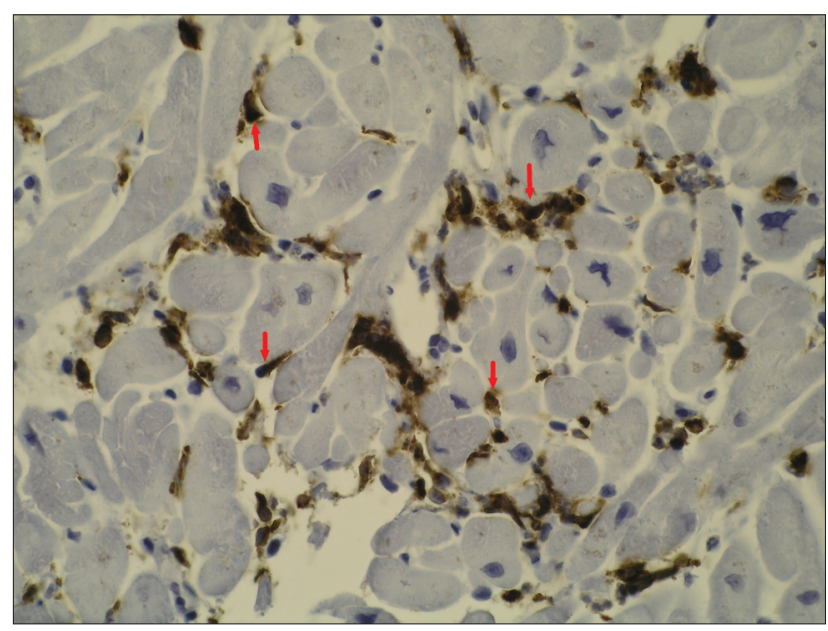

Fig. 1. LCA+ cells significant inflammatory infiltration. cardiogenic shock. She was transferred to our department, on admission with hypotension and sinus tachycardia; a chest X-ray indicated congestion and dilatation of the cardiac shadow. A control echocardiographic examination demonstrated continuous LV dysfunction; MRI confirmed this finding and showed possible inflammatory changes in the LV myocardium. EMB was performed; the samples had elevated numbers of infiltrating leukocytes (LCA+ cells) and T-lymphocytes (CD3+ cells): 27 LCA+ cells $/ \mathrm{mm}^{2}$ and $8 \mathrm{CD} 3+$ cells $/ \mathrm{mm}^{2}$ (Fig. 1). Polymerase chain reaction (PCR) method demonstrated no viral genome in the myocardium. We diagnostically concluded peripartum cardiomyopathy on the basis of myocarditis.

In light of the unimproved clinical status and the echocardiographic findings, immunosuppressive therapy (azathioprine + prednisone combination) was initiated. The patient's situation was gradually stabilized, and her therapy was converted to oral medication. Echocardiographic control 3 months after the definitive diagnosis (at the end of immunosuppressive therapy) showed only a slight improvement (LVEF increased to $20-25 \%$, persistent LV dilatation).

A control EMB was repeated 6 months after the initial one. The result showed a resolved inflammation with a decline in the number of infiltrating cells ( 8 LCA+ cells/ $\mathrm{mm}^{2}$ and $0 \mathrm{CD} 3+$ cells $/ \mathrm{mm}^{2}$, see Fig. 2), but without major changes in LV function or morphology. A cardioverter defibrillator (ICD) was subsequently implanted as a primary prevention of sudden cardiac death, due to the narrow QRS complexes and the absence of contraction asynchrony without resynchronization function. The patient was followed as a potential candidate for heart transplantation, clinically in NYHA functional class III. Gradual progression of fatigue was observed one year after delivery, with decreased exercise tolerance and increased breathlessness. A right heart catheterization control revealed significant reduction in cardiac output and rise in mean pulmonary artery and wedge pressures, further elevation of transpulmonary gradient (TPG) and pulmonary vascular resistance (PVR) with good response to the vasodilator test. An echocardiographic examina-

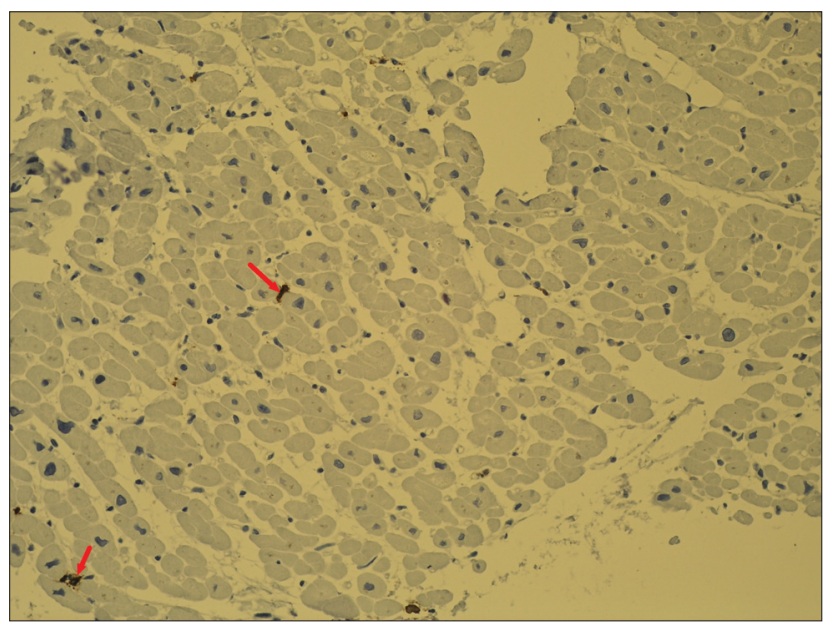

Fig. 2. LCA+ cells nonsignificant residual inflammatory infiltration. 
tion showed dysfunction and dilatation of LV, with EF $20 \%$, with more pronounced mitral regurgitation, right ventricle $(\mathrm{RV})$ dilatation, and pulmonary hypertension than in the previous examinations. The patient was indicated for heart transplantation and put on a waiting list.

\section{CLINICAL CASE 2}

A 37 year old woman in the 36th week of her second pregnancy ( 1 fetus) experienced a respiratory tract infection treated with orally administered antibiotics. After temporary improvement, repeated worsening of breathlessness with shading in the pulmonary parenchyma on $\mathrm{X}$-ray was diagnosed as bronchopneumonia. The patient was hospitalized for intravenous antibiotic treatment. On the third day after admission to the pneumology department, the patient observed the absence of fetal movements. An immediate gynecological inspection noted fetal death; the fetus was subsequently delivered per vias naturales. After discharge from the hospital, the patient was stable for one week, but then experienced increased shortness of breath and weakness. Computed tomography angiography (CTA) was performed to explore suspected pulmonary embolism, but this diagnosis was excluded as the cause of difficulties. Echocardiography was indicated due to ongoing resting dyspnea, hyposaturation, and tachycardia. Severe LV dysfunction without dilatation was revealed (diastolic diameter $42 \mathrm{~mm}$, LVEF around 20\%). Pharmacological treatment for heart failure was initiated and transfer to our department was arranged. An echocardiographic control 2 weeks after the introduction of treatment showed only a slight improvement in LV function with EF 30\%, without dilatation of both ventricles. EMB was performed, with a finding of significant inflammatory infiltration in the myocardium (25 LCA+ cells/ $\mathrm{mm}^{2}, 14 \mathrm{CD} 3+$ cells $\left./ \mathrm{mm}^{2}\right)$. The presence of parvovirus B19 (PVB19) in the myocardium was detected by PCR method. The diagnostic conclusion was peripartum cardiomyopathy on the basis of myocarditis.

Due to the presence of a viral genome in the myocardium, immunosuppressive therapy was not indicated and standard heart failure treatment was continued. The patient was released in a clinically stable state (NYHA functional class II) for ambulatory care. Significant improvement in LV function to EF 50\% was demonstrated at the 3-month follow-up visit.

A previously-scheduled control EMB at the 6-month follow-up visit showed a significant regression of inflammation to below-the-limit cell numbers (10 LCA+ cells/ $\mathrm{mm}^{2}, 3 \mathrm{CD} 3+$ cells $/ \mathrm{mm}^{2}$ ). A normal echocardiographic finding was seen one year after the beginning of the disorder, LVEF was around $60 \%$, and the patient was subjectively without health complaints.

\section{CLINICAL CASE 3}

A 34 year old woman in the first month after a second uncomplicated pregnancy completed by the spontaneous birth of her second child observed gradually increasing inefficiency, fatigue, and shortness of breath. In the 4th month after the birth, the symptoms had progressed to resting breathlessness, only just at that point she visited her physician. She was sent to the hospital. CTA excluded pulmonary embolism, echocardiography captured heart failure with severe LV dysfunction, EF close 20\%, and LV dilatation with diastolic diameter $65 \mathrm{~mm}$. Lactation was suppressed with 14 days bromocriptine administration, and standard heart failure treatment was introduced. At that time, an MRI revealed multiple patchy deposits of late gadolinium enhancement, which corresponded to a possible inflammatory involvement of LV walls. The clinical status was stabilized at NYHA class II, some improvement in LV function was seen (LVEF 30-35\%). EMB was recommended; marked inflammatory infiltration was detected in myocardial samples (31 LCA+ cells/ $\mathrm{mm}^{2}, 15 \mathrm{CD} 3+$ cells $/ \mathrm{mm}^{2}$ ), PCR method did not detect any genome of potential etiological agents in the myocardium. The case was diagnosed as PPCM on the basis of myocarditis.

Three months of immunosuppressive therapy (prednisone + azathioprine) was initiated. An echocardiographic control after this treatment showed no improvement, with LVEF of $30 \%$ and a slight progression of LV dilatation. The patient was clinically stable in NYHA class II. Over the next 3 months (6 months after diagnosis), a control EMB showed a decline in inflammatory infiltration, but still meeting the criteria for ongoing myocarditis with signs of healing (16 LCA + cells $/ \mathrm{mm}^{2}, 8 \mathrm{CD} 3+$ cells $/ \mathrm{mm}^{2}$ ), LVEF at the time was $35-40 \%$, NYHA I-II. At the next follow-up visit 12 months after diagnosis, deterioration in clinical status (NYHA II-III) was noted, accompanied by worsening of echocardiographic findings. LVEF dropped to $30 \%$, with persistent dilatation of both ventricles (Fig. 3). Another EMB was not indicated, the pharmacological treatment of heart failure was optimized. During Holter ECG monitoring were captured a

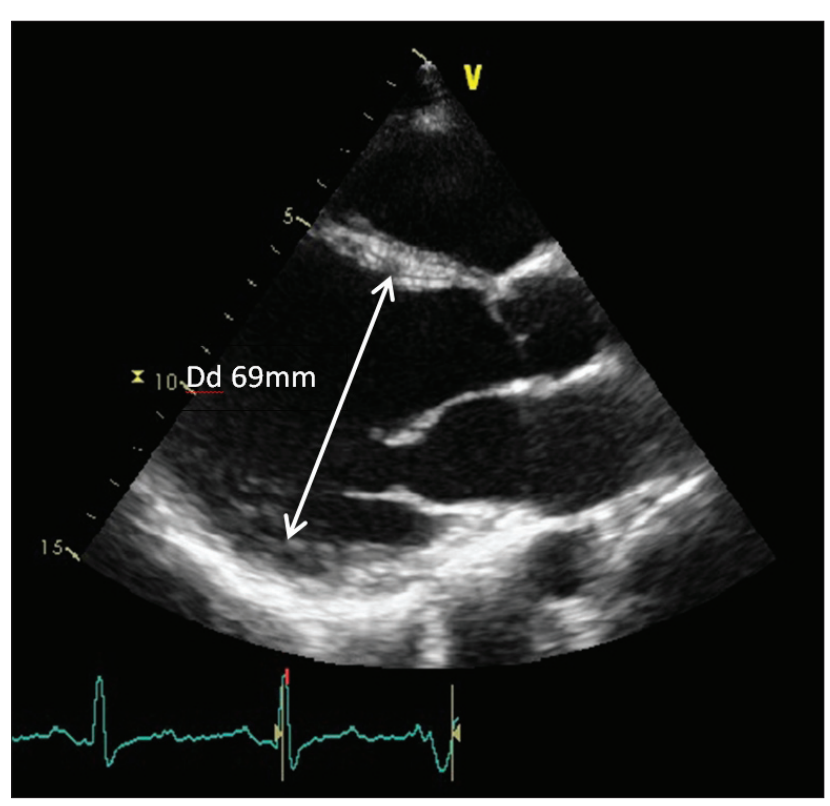

Fig. 3. Echocardiographic finding. 
few runs of nonsustained ventricular tachycardia up to 10 QRS complexes. A biventricular ICD implantation was strongly recommended (LBBB, QRS width $130 \mathrm{~ms}$ ), but was repeatedly refused by the patient. Eight weeks later, she died suddenly during sleep.

\section{DISCUSSION}

In Europe, PPCM is a rare disease although data specifically from this region are lacking. Most of the information we have is from the U.S.and South Africa.

The PPCM cases presented here show some common features of the disorder, but also some differences. Most cases manifest in the postpartum period. It is interesting that the patient (case 1) diagnosed during pregnancy would not have met the older diagnostic criteria because the symptoms began before the last month of pregnancy. The worsening of a pre-existing heart disease, most likely dilated cardiomyopathy (DCM), should be considered in the differential diagnosis for this case. This deterioration of pre-existing DCM, however, is usually seen a bit earlier in the pregnancy, most often in the second trimester when the greatest hemodynamic load is present ${ }^{10}$. However, the previous history of viral infection and the biopsy finding of significant inflammatory cell infiltration (myocarditis) somewhat contradict this theory.

Although the etiology of PPCM is still not clear, myocarditis is one of the longest and most suspected causes $^{4,16-18}$. Indeed, the presence of myocarditis was a common feature of all three cases. As early as the late 1960 s, a mouse model demonstrated that pregnant animals have a higher susceptibility to viral infection and that viruses replicate more in the myocardium of pregnant animals ${ }^{10}$. Similarly, hormone-dependent immunological changes were found in pregnant women that may lead to increased susceptibility to viruses and to more severe courses of such infections ${ }^{17}$.

Cardiotropic viruses are the most typical cause of "common" myocarditis in non-pregnant individuals. Several studies have been conducted in which EMBs were performed on women with PPCM in the past, and myocardial inflammation was detected in a wide range of them (8.8-78\%). The highest incidence of myocarditis was captured in a study by Midei et al, who performed EMBs on 18 patients with severe LV dysfunction (mean LVEF $22 \%$ ) over a 5 -year period ${ }^{18}$. These EMBs were evaluated according to Dallas criteria (i.e., only histologically), which have a much lower sensitivity than the immunohistological evaluations used today. Yet almost $80 \%$ of the affected women in this study had evidence of myocarditis. As in studies of common myocarditis, patients with PPCM had high titers of auto-antibodies against a number of myocardial antigens, as well as increased levels of proinflammatory cytokines showing the abnormal immune and inflammatory response in these patients ${ }^{10,16}$.

The second clinical case in our study confirms the above-mentioned experience that in patients without significant LV dilatation at the beginning there is a better chance of LV function restoration. Also, early improve- ment in LV function is a positive indicator of favorable conditions in the future. This is not entirely confirmed by the third case in our study, when echocardiographic variations progressing to recurrent LV dysfunction were found after initial improvement. In this case, however, LV dilatation was present at diagnosis, unlike the second case. The first and third patients, in accordance with the EMB results, were treated with immunosuppressive combination therapy. In addition to the studies published by Midei ${ }^{18}$ and Melvin ${ }^{19}$ of PPCM patients, the effectiveness of such therapy was demonstrated in patients with "nonpregnancy-associated myocarditis" in two randomized trials by Wojnic ${ }^{20}$ and Frustaci ${ }^{21}$. In both of our patients treated with immunosuppressive therapy, the number of infiltrating inflammatory cells significantly decreased, but the effect on the improvement of LV function was not entirely convincing. In the first case, there was only a small improvement in systolic function without a reduction in the size of LV; in the third case, the effect was initially more pronounced, but LV dysfunction later recurred. In addition, it must be said that the decline in the number of infiltrating cells was also evident in the natural course of the disease illustrated in the second case in our study, which was not treated with immunosuppressive therapy. These results suggest that the effect of immunosuppressive therapy is at least ambiguous.

A current etiopathogenetic theory is the concept of increased oxidative stress in pregnancy as the cause of the development of PPCM. Oxidative stress is a trigger of cathepsin D activation. Cathepsin D is a protease that cleaves the nursing-hormone prolactin into the $16 \mathrm{kDa}$ prolactin with angiostatic and proapoptotic effects that also induces vasoconstriction and damages the function of myocytes ${ }^{22}$. Experimental data from mouse models show that the defect in the antioxidant mechanisms can lead to the development of PPCM. The role of oxidative stress - cathepsin D - prolactin - $16 \mathrm{kDa}$ prolactin cascade in the etiology of PPCM is supported by the fact that the administration of the dopamine D2-receptor agonist bromocriptine, which blocks the production of prolactin, leads to the prevention of PPCM in mice ${ }^{22,23}$.

This therapeutic concept has been tested in the first pilot study in humans, which suggested a possible beneficial effect $^{23}$. The third clinical case in our study was also given bromocriptine, but the treatment duration was shorter than in the pilot study by Sliwa et al. In the treatment of our patient, we observed only a slight improvement in echocardiographic findings. One of the key pathways where the different pathophysiological mechanisms might interact could be apoptosis. In addition to its role in oxidative stress-induced myocardial damage, apoptosis is thought to participate in inflammatory myocardial involvement. Women with PPCM have presented with increased levels of markers of apoptosis and inflammation (Fas/Apo1, TNF-alpha and CRP) $\left(\right.$ ref. $\left.^{3}\right)$.

The data on therapy for PPCM are limited, and evidence for the treatment of this disorder is quite uncertain. We transmit the experience from standard systolic heart failure therapy to PPCM without confirmation of such therapy effect in this special group of patients. Treatment 
during pregnancy should be modified with regard to possible birth defects related to the medication. Before delivery, it is necessary to remove angiotensin converting enzyme inhibitors (ACE-I) and angiotensin receptor blockers (ARB) from standard systolic heart failure medication. They should be replaced with hydralazine, nitrates, or their combination. It is advisable to use preferentially cardioselective beta-blockers, and low dose diuretics; in critical cases dobutamine, dopamine, milrinone, or nitrates can be used. ACE-I / ARB, beta blockers, spironolactone, digoxin, diuretics, or anticoagulant therapy can be administered even during breast-feeding ${ }^{10}$.

Besides this "classical" heart failure treatment, experimental and small clinical studies have tested drugs with quite optimistic results, but independent confirmation is still pending. This includes pentoxifylline, a drug reducing the production of TNF-alpha and preventing apoptosis, which despite the positive results of one study has not spread more widely ${ }^{24}$.

The previously mentioned drug bromocriptine, a prolactin inhibitor used for years to suppress lactation, is currently often discussed. Its administration stops oxidative stress-induced cleavage of prolactin by protease cathepsin $\mathrm{D}$ to $16 \mathrm{kDa}$ prolactin subfragment, which has angiostatic and pro-apoptotic effect and is associated with inflammatory response in vascular endothelium, altered metabolism of myocytes, and impaired myocardial contractility. Its effect has been studied with promising results in animal models and also in a pilot proof-of-concept study of 20 women $^{23}$.

The use of immunosuppressive therapy is offered as a parallel to studies with inflammatory cardiomyopathy in patients with biopsy-proven myocarditis, where a viral (or other) genome is not present in the myocardium ${ }^{20,21}$. There are only limited data from patients with PPCM. In the above cited study by Midei et al, myocarditis was demonstrated in 14 out of 18 patients; 10 of these 14 patients were treated with immunosuppressive combination azathioprine + prednisone. Control echocardiographic examination after 6-8 weeks showed statistically significant improvement in LV function along with decrease of LV size, which contrasted with the group without evidence of inflammation where no improvement was seen ${ }^{18}$.

It should be emphasized that any immunosuppressive therapy consideration is absolutely dependent on the performance of EMB and the knowledge of its results, including immunohistological and molecular genetic testing of samples. In cases without proven myocarditis or with the presence of pathological agents in the myocardium, such treatment cannot be indicated ${ }^{25}$.

When deciding whether non-pharmacological treatment is indicated, it should be kept in mind that up to $50 \%$ of patients could improve LV function within 6 months from the onset of symptoms ${ }^{15}$. Restraint should be applied in the indication of early implantation of an implantable cardioverter defibrillator (ICD) with or without resynchronization function (CRT). But as our third case showed, too long hesitation, especially in the presence of serious result of ECG Holter monitoring, can be fatal. Left ventricular or biventricular mechanical circulatory support can serve as a "bridge to recovery" or "bridge to transplantation" in the cases with most severe heart failure. In such situations, heart transplantation should be also considered as the last treatment option for refractory heart failure not responding to standard therapies $^{1,9}$. In our small group of patients with PPCM, one patient is currently included on the waiting list for heart transplantation and is also treated with ICD at the same time. Another patient was indicated for implantation of CRT/ ICD in primary prevention of sudden cardiac death, but the patient decided to postpone implantation for a few more months in the hopes of improvement. Unfortunately, she died suddenly, most likely due to malignant arrhythmias. Discussion about the possibilities of further pregnancies is inevitable with this disorder. In subsequent pregnancies, women with a history of PPCM are at high risk of re-deterioration of LV systolic function and recurrence of heart failure symptoms, even patients with normalization of LV function in the meantime ${ }^{1,26}$. Persistent LV dysfunctions as well as severe impairment from the previous pregnancy (LVEF below 25\%) are considered to be a clear contraindication for further pregnancies. Another pregnancy is not recommended, even for women who develop significant improvement or even normalization of left ventricular function after previous PPCM (ref. ${ }^{1,3,4,9,10}$ ).

\section{CONCLUSION}

PPCM is a disorder with unclear causes and clinical course. Despite many recent attempts, there is not yet a universal therapeutic approach if such can exist at all. As shown in the described cases, the pursuit of targeted therapy based on the use of a range of modern diagnostic methods including EMB may not be successful, and some patients become candidates for device therapy or heart transplantation. In contrast, in a percentage of cases the clinical situation and echocardiographic examination results improve but we can never be certain this is the result of our treatment strategies or whether it is a natural course of the disease. Sufficiently robust data providing more accurate and more successful treatment for a rare disorder like PPCM requires broad-based multicenter randomized trials.

\section{ACKNOWLEDGEMENT}

Supported by European Regional Development Fund - Project FNUSA-ICRC (No. CZ.1.05/1.1.00/02.0123).

\section{CONFLICT OF INTEREST STATEMENT}

None declared. 


\section{REFERENCES}

1. Sliwa K, Hilfiker-Kleiner D, Petrie MC, Mebazaa A, Pieske B, Buchmann E, Regitz-Zagrosek V, Schaufelberger M, Tavazzi L, van Veldhuisen DJ, Waktins H, Shah A, Seferovic PM, Elkayam U, Pankuweit S, Papp Z, Mouquet F, McMurray JV. Current state of knowledge on aetiology, diagnosis, management, and therapy of peripartum cardiomyopathy: a position statement from the Heart Failure Association of the European Society of Cardiology Working Group on peripartum cardiomyopathy. Eur J Heart Fail 2010;12:767-78.

2. Pearson GD, Veille JC, Rahimtoola S, Hsia J, Oakley CM, Hosenpud JD, Ansari A, Baughman KL. Peripartum cardiomyopathy: National Heart, Lung, and Blood Institute and Office of Rare Diseases (National institutes of Health) workshop recommendations and review. JAMA 2000;283:1183-8.

3. Ramaraj R, Sorrell VL. Peripartum cardiomyopathy: Causes, diagnosis, and treatment. Cleve Clin J Med 2009;76(5):289-96.

4. Sliwa K, Fett J, Elkayam U. Peripartum cardiomyopathy. Lancet 2006;368:687-93.

5. Gentry MB, Dias JK, Luis A, Patel R, Thornton J, Reed GL. AfricanAmerican women have a higher risk for developing peripartum cardiomyopathy. J Am Coll Cardiol 2010;55:654-9.

6. Elliott $P$, Andersson B, Arbustini E, Bilinska Z, Cecchi F, Charron $P$ Dubourg O, Kühl U, Maisch B, McKenna WJ, Monserrat L, Pankuweit S, Rapezzi C, Seferovic P, Tavazzi L, Keren A. Classification of the cardiomyopathies: a position statement from the European Society of Cardiology Working Group on Myocardial and Pericardial Diseases. Eur Heart J 2008;29:270-6.

7. Maron BJ, Towbin JA, Thiene G, Antzelevitch C, Corrado D, Arnett D, Moss AJ, Seidman CE, Young GB. Contemporary definitions and classification of the cardiomyopathies: An American Heart Association Scientific Statement from the Council on Clinical Cardiology, Heart Failure and Transplantation Committee; Quality of Care and Outcomes Research and Functional Genomics and Translational Biology Interdisciplinary Working Groups; and Counci on Epidemiology and Prevention. Circulation 2006;113:1807-16.

8. Van Spaendonck-Zwarts KY, Van Tintelen JP, van Veldhuisen DJ, van der Werf R, Jongbloed JDH, Paulus WJ, Dooijes D, van den Berg M Peripartum cardiomyopathy as a part of familial dilated cardiomyopathy. Circulation 2010;121:2169-75.

9. Elkayam U. Clinical characteristics of peripartum cardiomyopathy in the United States. Diagnosis, prognosis, and management. J Am Coll Cardiol 2011;58:659-70.

10. Abboud J, Murad Y, Chen-Scarabelli C, Saravolatz L, Scarabelli TM. Peripartum cardiomyopathy: A comprehensive review. Int J Cardio 2007:118:295-303.

11. Nabhan A. Peripartum cardiomyopathy. ASJOG 2005;2:231-7.

12. Schultheiss HP, Kühl U, Cooper LT. The management of myocarditis. Eur Heart J 2011;32:2616-25.
13. Cooper LT, Baughman KL, Feldman AM, Frustaci A, Jessup M, Kühl U, Levine GN, Narula J, Starling RC, Towbin J, Virmani R. The role of endomyocardial biopsy in the management of cardiovascular disease: a scientific statement from the American Heart Association, the American College of Cardiology, and the European Society of Cardiology. Circulation 2007;116:2216-33.

14. Narula N, McNamara DM. Endomyocardial biopsy and natural history of myocarditis. Heart Fail Clin 2005;1:391-406.

15. Cooper LT, Mather PJ, Alexis JD, Pauly DF, Torre-Amione G, Wittstein IS, Dec GW, Zucker M, Narula J, Kip K, McNamara DM. Myocardial Recovery in Peripartum Cardiomyopathy: Prospective Comparison With Recent Onset Cardiomyopathy in Men and Nonperipartum Women. J Cardiac Fail 2012;18:28-33.

16. Lamparter S, Pankuweit S, Maisch B. Clinical and immunologic characteristics in peripartum cardiomyopathy. Int J Cardiol 2007;118:1420.

17. Bültmann BD, Klingel K, Näbauer M, Wallwiener D, Kandolf R. High prevalence of viral genomes and inflammation in peripartum cardiomyopathy. Am J Obstet Gynecol 2005;193:363-5.

18. Midei MG, DeMent SH, Feldman AM, Hutchins GM, Baughman $\mathrm{KL}$. Peripartum myocarditis and cardiomyopathy. Circulation 1990;81:922-8

19. Melvin K, Richardson P, Olsen EG, Daly K, Jackson G. Peripartum cardiomyopathy due to myocarditis. N Engl J Med 1982;307:731-4.

20. Wojnicz R, Nowalany-Kozielska E, Wojciechowska C, Glanowska G, Wilczewski P, Niklewski T, Zembala M, Polonski L, Rozek MM, Wodniecki J. Randomized, placebo-controlled study for immunosuppressive treatment of inflammatory dilated cardiomyopathy: two-year follow-up results. Circulation 2001;104:39-45.

21. Frustaci $A$, Russo MA, Chimenti $C$. Randomized study on the ef ficacy of immunosuppressive therapy in patients with virus-negative inflammatory cardiomyopathy: the TIMIC study. Eur Heart J 2009;30:1995-2002

22. Hilfiker-Kleiner D, Sliwa K, Drexler H. Peripartum cardiomyopathy: Recent insights in its pathophysiology. Trends Cardiovasc Med 2008;18:173-9.

23. Sliwa K, Blauwet L, Tibazarwa K, Libhaber E, Smedema JP, Becker A, McMurray J, Yamac H, labidi S, Struman I, Hilfiker-Kleiner D. Evaluation of bromocriptin in the treatment of acute severe peripartum cardiomyopathy: a proof-of-concept pilot study. Circulation 2010;121:1465-73.

24. Sliwa K, Skudicky D, Candy G, Bergemann A, Hopley M, Sareli P. The addition of pentoxiphylline to conventional therapy improves outcome in patients with peripartum cardiomyopathy. Eur J Hear Fail 2002;4:305-9.

25. Sliwa K, Fett J, Elkayam U. Peripartum cardiomyopathy. Lancet 2006;368:687-93

26. Elkayam U, Tummala PP, Rao K, Akhter MW, Karaalp IS, Wani OR, Hameed A, Gviazda I, Shotan A. Maternal and fetal outcomes of subsequent pregnancies in women with peripartum cardiomyopathy. N Engl J Med 2001;344:1567-71. 